\title{
Neural network models in reducing the risks of irrigated agriculture
}

\author{
Galina Kamyshova ${ }^{1,1,}$ \\ ${ }^{1}$ Saratov State Agrarian University, Theatralnaia pl., 1, Saratov, 410012, Russia
}

\begin{abstract}
The article presents the results of studies on the construction of neural network models that will help reduce risks and increase the efficiency of irrigated agriculture. The projected increase in food production on irrigated land is subject to significant risks of climatic and infrastructural nature. Irrigated agriculture, in the global understanding of interrelationships, is a complex dynamic system with nonlinear dependencies. Therefore, traditional approaches based only on physical modeling of environmental and technical processes and relationships often complicate the search for effective solutions. Technological advances that stimulate unprecedented data growth, the rapid extraction of meaningful information from the modern data flow can increase the efficiency of decisions and minimize risks. An approach based on the implementation of neural network models for predicting agro-climatic parameters and intelligent control of irrigation equipment using neurocontrollers is proposed. The models are implemented in the Matlab. The use of these models can significantly reduce risks and increase the efficiency of irrigated agriculture.
\end{abstract}

\section{Introduction}

Agriculture plays a significant role in the global economy due to the global challenges we face. The world's population is growing at a significant rate; according to UN's forecasts by 2050 it will reach 10 billion people. As a result, the need for food may increase by more than 1.7 times. The key factors influencing the development of agriculture include the need for food, the availability of resources (land and water), as well as natural and climatic capabilities. Over the past 50 years, agricultural production on a global scale has grown 2.53 times, and the growth of acreage has been about $12 \%$. According to forecasts, the increase in production will occur due to its intensification on existing arable land. At the same time, the role of irrigation is increasing, since more than $40 \%$ of the growth in world food production and $60 \%$ of grain production falls on irrigated lands. Currently, world agriculture uses $12 \%$ of the land surface and $70 \%$ of water for crop production. According to forecasts, by 2050 , the area of irrigated land will increase by $6 \%$, the volume of water withdrawn for irrigation will increase by about $10 \%$. An increase in food production on

${ }^{1}$ Corresponding author: gkamichova@mail.ru 
irrigated land is predicted by $38 \%$ due to the expected increase in the intensity of farming and an increase in productivity [1]. At the same time, the rate of expansion of the area of irrigated land, which in the middle of the last century was over $2 \%$ per year, has significantly decreased. Growing water scarcity limits the ability to produce food on irrigated land, especially in the countries and regions most affected by it. The amount of water withdrawn varies greatly from country to country and region to region. Europe consumes only $6 \%$ of its domestic resources; of these, only $29 \%$ go to the needs of agriculture. Asian economies consume $20 \%$ of their domestic renewable resources, of which more than $80 \%$ goes to irrigation. In many areas of the Middle East, North Africa and Central Asia with low rainfall, most of the usable water $(80-90 \%)$ is used for agriculture [2]. The second important component is the technical equipment of irrigated agriculture, which in many countries is at a rather low level. In this regard, as well as in light of the problem of global climate change, the problem of reducing the risks of irrigated agriculture in general and its individual components in particular is urgent.

Irrigated agriculture, in the global understanding of interrelationships, is a complex dynamic natural and technical system with nonlinear dependencies. Therefore, traditional approaches based only on physical modeling of natural and technical processes and relationships often complicate the search for effective solutions. Technological advances that stimulate unprecedented data growth, the rapid extraction of meaningful information from the modern data flow can increase the efficiency of decisions and minimize risks. Along with this, new tools for data mining, deep learning provide improved accuracy by resolving complex relationships in large volumes of natural and synthetic parameters and have great potential.

In recent years, much research has focused on the application of data mining and deep learning techniques to improve irrigation efficiency and reducing the risks. Thus, McCarthy [3] has developed a cotton irrigation management system, Navarro-Hel [4], Giusti [5] decision support systems for irrigation management. New approaches and tools in predicting climatic parameters were presented in the works S. Rasp [6], F. Sévellec [7].

In this regard, the presented study is focused on building neural network models that will help reduce risks and increase the efficiency of irrigated agriculture.

\section{Materials and Methods}

\subsection{Problem statement}

Irrigated agriculture belongs to the highly intensive areas of agricultural production. The efficiency of the functioning of irrigated agriculture is influenced by many different factors, among which two key ones can be distinguished - these are environmental and technical.

The most important environmental problem facing humanity in the new 21 st century is the rise in temperature of the climate system of our planet - the so-called global warming. So, for example, over the past thirty years on the territory of the Saratov region of Russia during the main growing season of grain crops (May-July) the climate aridity increased: the average air temperature increased by $0.80 \mathrm{C}$, and the amount of precipitation decreased by $10 \mathrm{~mm}$, which negatively affects the yield spring crops. The amount of precipitation in August decreased, which often worsens the conditions for obtaining seedlings of winter wheat, but at the same time, the amount of autumn-winter precipitation (NovemberFebruary) increased by $20 \%$, thereby improving the conditions for its wintering [8]. That is, agro-climatic factors have a significant impact on agricultural production, especially in the zones of the so-called risky farming. They largely determine the size and quality of the crop, as well as the characteristics of agro-technical measures for their cultivation. On the other hand, in conditions of stochastic variability of agro-climatic resources, the technical 
component, namely, irrigation machines should develop towards improving the accuracy of irrigation, since the ineffective operation of sprinkler equipment leads to significant environmental and economic consequences. In this regard, the diagram of the relationship between the risks of irrigated agriculture can be represented as the interaction of environmental and technical risks (figure 1).
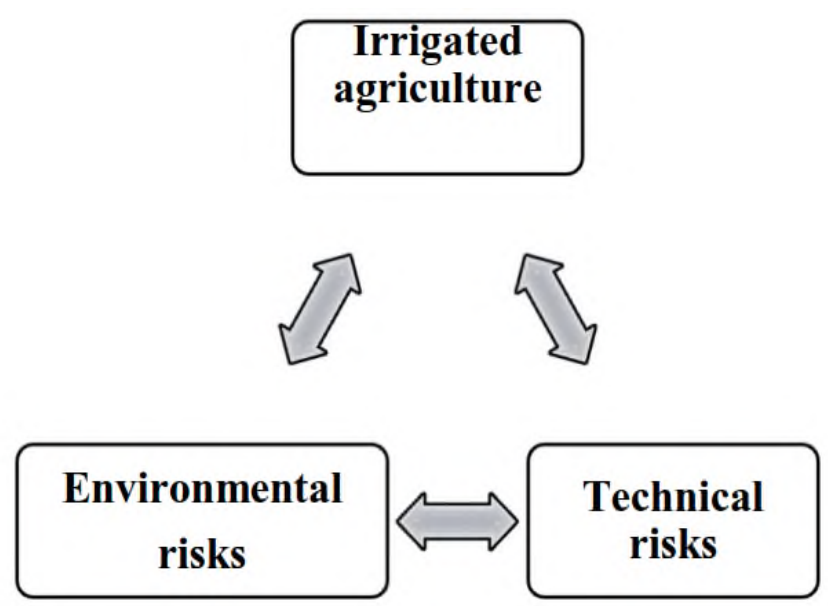

Fig. 1. The system of interaction

The system of interaction "Environmental risks - Technical risks" is a complex system whose behavior and properties are not the sum of the properties of individual components.

The mathematical model of such a system can be represented in the form of a functional relationship between the variables of the current states of technical risks - $X(t)$, the current states of natural risks $-Z(t)$, control actions - $\mathrm{U}(t)$, and the actual response $Y(t)$ of the system to these exposures (cumulative exposure to risk) at a certain point in time t:

$$
Y(t)=F(X(t), Z(t), U(t)) \text {. }
$$

$X(t)=\left\{X_{\mathrm{i}}\left(t_{\mathrm{j}}\right)\right\}$ - is a vector, where $X_{\mathrm{i}}\left(t_{\mathrm{j}}\right)$ is the $\mathrm{i}$-th state of the technical risk system at time $t_{\mathrm{j}}$

$Z(t)=\left\{Z_{\mathrm{i}}\left(t_{\mathrm{j}}\right)\right\}$ - is a vector, where $Z_{\mathrm{i}}\left(t_{\mathrm{j}}\right)$ is the $\mathrm{i}$-th state of the natural risk system at time $t_{\mathrm{j}}$;

$U(t)=\left\{U_{\mathrm{i}}\left(t_{\mathrm{j}}\right)\right\}$ - is a vector, where $U_{\mathrm{i}}\left(t_{\mathrm{j}}\right)$ is the $\mathrm{i}$-th control action on the system at time $t_{\mathrm{j}}$.

The task is to find such control actions $\mathrm{U}(t)$, which will minimize $Y(t)$.

To solve this problem, it is proposed to use artificial neural networks as universal approximators of functions capable of displaying any complex nonlinear dependence and can intelligently study these functions in the learning process. The ANN's ability to display sets of input / output data with an acceptable range of errors makes them indispensable tools for modeling natural and technical processes.

\subsection{Description of the method and materials}

Artificial neural networks (ANN) are increasingly used in various applied problems [9, 10]. The main element of a neural network is a neuron. An artificial neuron is the simplest analog transforming element that models the basic ideas about the work of a living neuron. 
Artificial neural networks are parallel computing devices consisting of many interacting simple processors (neurons).

The mathematical formalization of an artificial neuron is the adder equation [11]

$$
s_{k}=\sum_{j=1}^{n} w_{j, k} \cdot x_{j}+b_{k}
$$

and activation block equation

$$
y_{k}=\phi\left(s_{k}\right)
$$

where, for $k$-th neuron, $x_{1}, x_{2}, \ldots, x_{n}$ - input signals; $w_{1, k}, w_{2, k}, \ldots, w_{n, k}$ - synoptic weights; $b_{k}$ - reference signal level; $s_{k}$ - linear adder output; $\phi\left(s_{k}\right)$ - activation block conversion function; $y_{k}$ - output signal.

A set of neurons connected by inputs and outputs makes up an artificial neural network. Artificial neural networks can have different architectures, which leads to different types of ANNs:

- static networks (direct distribution), in which the graphs do not have loops;

- dynamic (recurrent) networks, or networks with feedback.

The simplest static network has a direct data transfer structure: data flows from inputs through hidden elements and eventually arrives at output elements. This structure has stable behavior. This is the simplest kind of neural network. Feedforward networks can be singlelayer or multi-layer (Figure 2 left). Dynamic neural networks (Figure 2 on right) are networks that apply branch delays or repeated connections. The component for including the initial and past states of the system is the reverse connection. This connection is repeated either from the output layer or from the hidden layer back to the context unit and after one time step returns to the input layer. Because dynamic networks have memory, they can be trained to learn sequential or time-varying patterns. In dynamic networks, the output depends not only on the current input to the network, but also on previous inputs, outputs, or states of the network. The simplest dynamic network is called Input Delay Neural Network (IDNN). This network consists of a multilayer neural network with a delay drop line at the input layer. Tapped delay lines can be viewed at all hidden layers of the network, which is called a time-delayed distributed neural network [12].
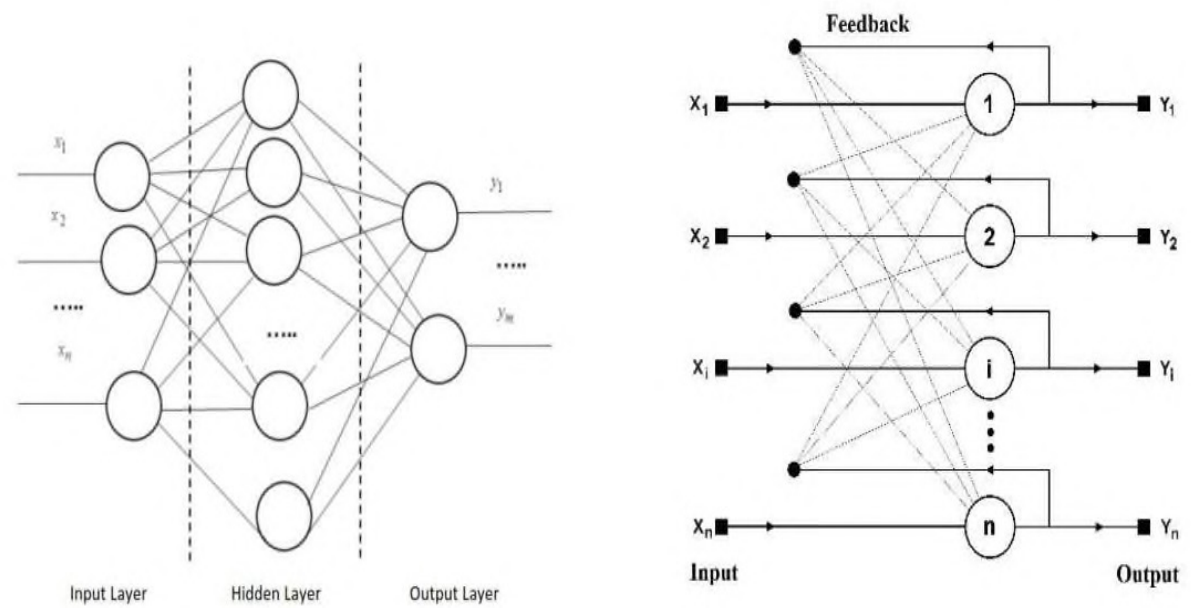

Fig. 2. Functional diagram of a simple multilayer neural network (left) and dynamic neural network (on right) 
We propose to use two neural network models that can reduce the natural and technical risks of irrigated agriculture. These models are based on both simple multilayer neural networks and dynamic neural networks. The technical risk reduction is based on the multilayer neural networks model, and the environmental risk reduction is based on the dynamic neural networks model.

As databases for model validation, we use data from official climate reference books, meteorological observations in the Roshydromet system, data from field experiments conducted on the basis of irrigation systems in the Saratov region of Russia, materials from the work of Russian scientists (for example, $[13,14]$.

\section{Results}

\section{Model 1.}

Multilayer neural networks can be used to identify and control irrigation systems.

The neural network acts as an adaptive regulator in the control system of sprinkler machines. In the learning process, an optimal control action is formed, which is fed to the input of the system.

The introduction of neurocontrol methods into control systems of sprinkler machines allows to improve the quality of functioning of complex systems with nonlinear objects and connections. Directly in the computational module of the system, a neurocontroller is synthesized, which for a given moment of time at known values of input disturbances, for example, velocities using a neural network, finds the value of the control action with its subsequent implementation to the control module. The task of neural network control is to bring the actual value of the irrigation rate as close as possible to the required one and optimize resources such as water and energy.

It is possible to use various software products, such as Matlab, R, Pyton, etc. for the synthesis of a neurocontroller [15] We have used an algorithm implemented in the Matlab environment (Neral NetworkToolbox package). At the first stage, the initial data were collected from the operating wide-grip sprinklers required for the synthesis of the neurocontroller. The task of synthesizing a neurocontroller is not trivial and requires meticulous work on the selection of the topology of neural networks, training parameters. Ultimately, a neural network should be obtained, which is a model of the controller, correctly generating the output signal on the training set. Based on the results of modeling on the test set, it can be concluded that the neuromodel produces an output signal corresponding to the expected signal, while the error (the difference between the required and the actual value of the irrigation rate) is minimal. The neural network must be able to convey the dynamics of the process. Then this network is connected to the regulator. The weights of the network are considered constant, and only the weights of the network regulator are adjusted, minimizing the discrepancy between the response of the reference model and the given neural networks to the same input signal; the neural network diagram and the convergence of the algorithm are shown in the figure (Fig. 3). 

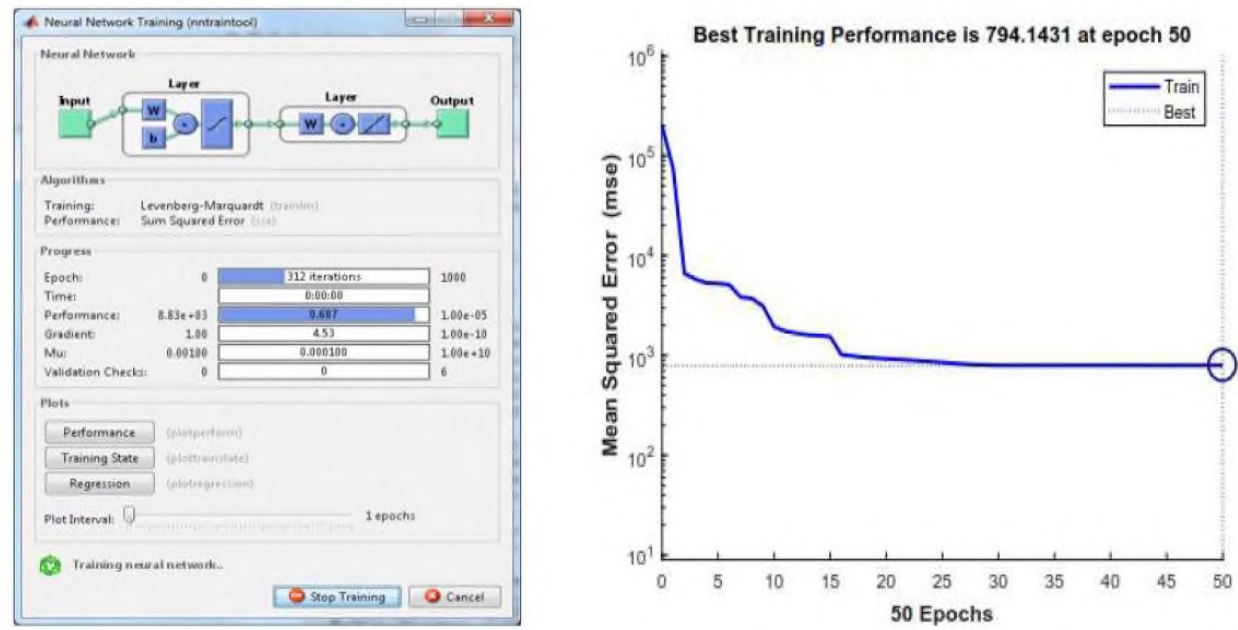

Fig. 3. Diagram of the training neuromodel (left) and convergence of the algorithm in Matlab

After learning, the regulator is used in the control loop, reducing deviations by up to 1$3 \%$. The model of neurocontrol by speed was investigated in [16], which also shows a functional control diagram with an integrated neuroregulator.

\section{Model 2:}

Dynamic Artificial Neural Networks are the best use of resources for the agro-climatic forecasting problems and can reduce the environmental risk [17]. We propose to consider a distributed neural network with a time delay (TDNN) as a method for predicting the agroclimatic resources of a region. The prediction parameters may be, for example, the sum of active temperatures above $10^{\circ} \mathrm{C}$ for the growing season, the moisture coefficient or average annual rainfall according to observation data. These parameters are included in many indicators characterizing the agro-climatic potential. The model is implemented in the Matlab system [18]. The network architecture as well as the learning algorithm and its convergence are shown in Figure 4.
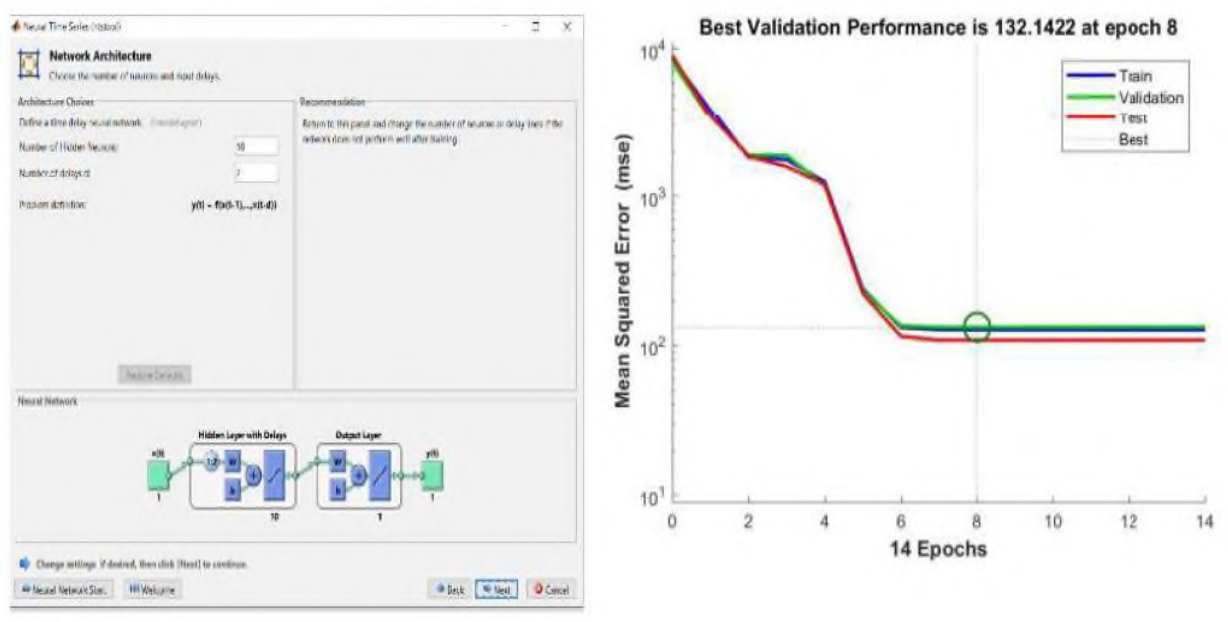

Fig. 4. Diagram of the learning algorithm (left) and its convergence in Matlab 
An analysis of numerical data shows that the average annual precipitation, for example, for the period under review, varied from 249 to $666 \mathrm{~mm}$. Agro-climatic resources change significantly over a 25 -year period, therefore the task of their accurate forecasting is very important. Due to significant fluctuations in values, even polynomial regression (Figure 5 top) is not able to achieve a high level of forecast accuracy. Having built a model for predicting the average annual precipitation of a distributed neural network with a time delay (TDNN) in the Matlab system, we get the following graph (Figure 5 bottom).

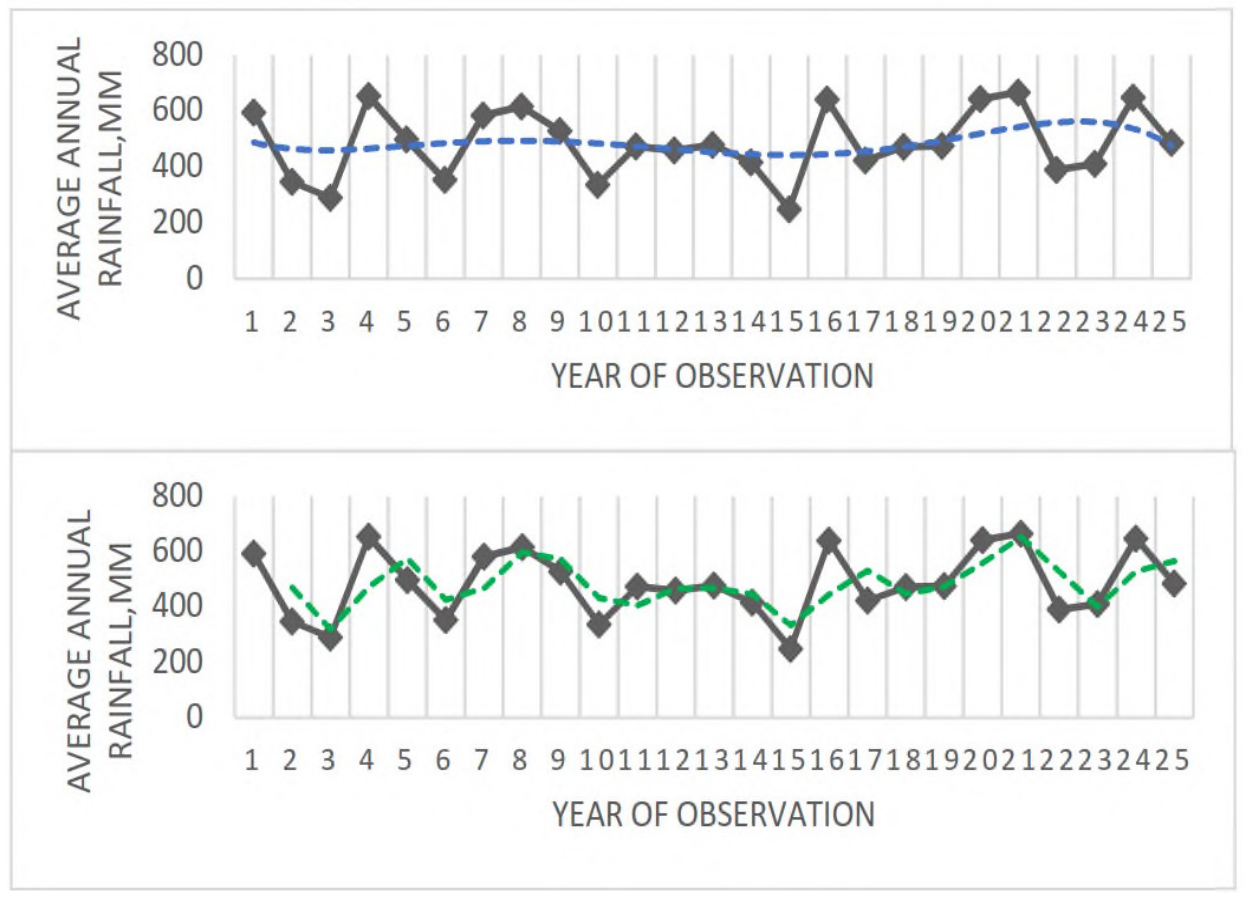

Fig. 5. Graphs of average annual precipitation (top) and its Matlab - simulations (bottom) - black line, polynomial regression - blue line, ANN predicted values - green line.

As can be seen from the graphs, the forecasting accuracy increases by an order of magnitude, which is especially important for reducing the risks of irrigated agriculture, which includes, for example, the Saratov region, since the loss of gross harvest in such years can be up to $50 \%$.

Thus, we propose the following algorithm for making management decisions based on neural networks that reduce risks in irrigated agriculture: using model 2, a general forecast of agro-climatic resources is constructed, then, on its basis, suitable crops and irrigation technologies are selected. The next step is to use Model 1 to operate the irrigation machine, which allows to reduce technical risks when directly irrigating agricultural fields. 


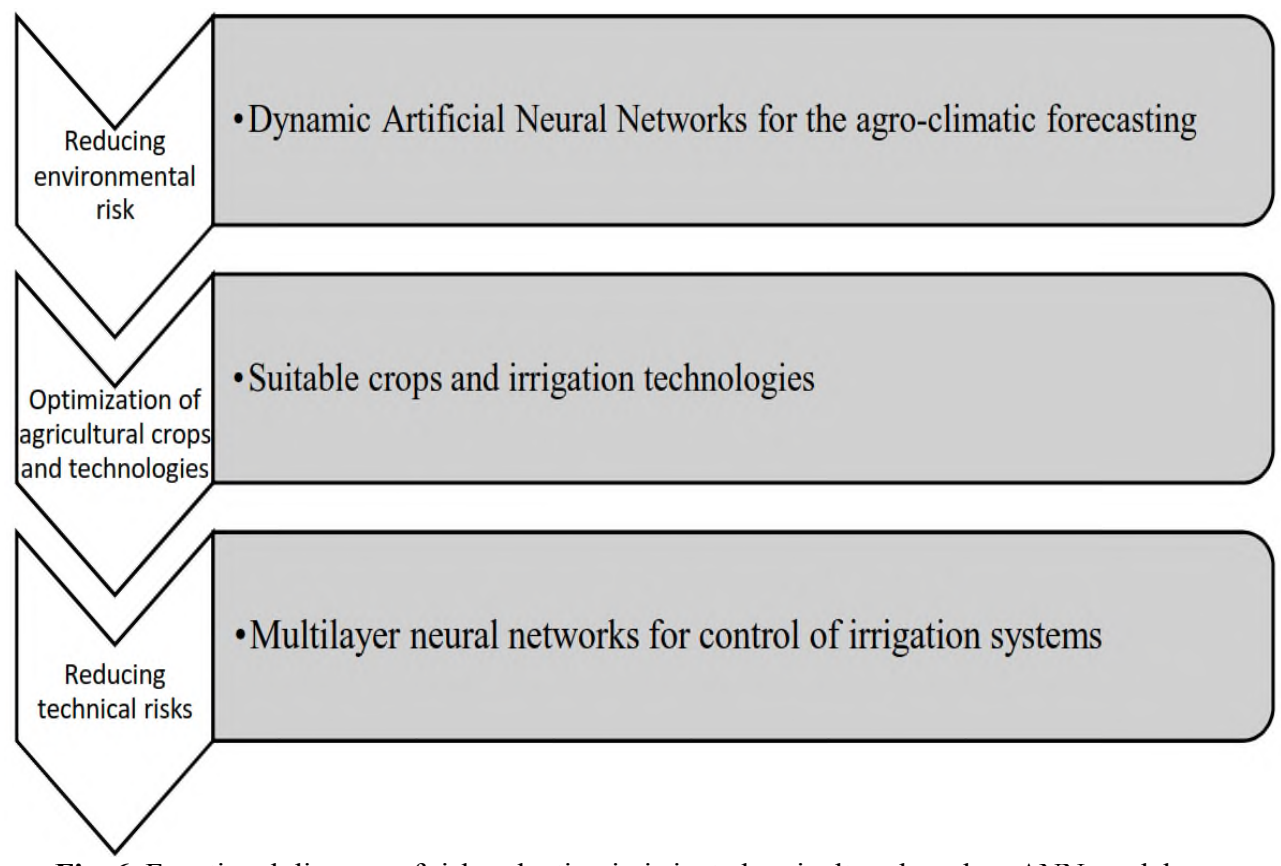

Fig. 6. Functional diagram of risk reduction in irrigated agriculture based on ANN models

\section{Conclusions}

Agro-climatic and technical factors have a significant impact on agricultural production, especially in areas of the so-called risky farming. They largely determine the size and quality of the crop, as well as the characteristics of agro-technical measures for their cultivation. Both during periods of intense droughts and favorable years for moisture, effective management is needed to reduce risks for the sustainable development of irrigated agriculture. It is necessary to plan irrigation and technical resources for their effective use, the correct selection of crops and varieties, the use of appropriate agricultural techniques, the rational placement of crops in accordance with their requirements for environmental conditions, and much more. The study proposes an approach to reduce the risks of irrigated agriculture based on artificial neural network models. The proposed models make it possible to reduce both environmental risks, building accurate neuro-forecasts of agroclimatic resources, and technical risks, by introducing neurocontrollers into irrigation systems.

The availability of modern software allows solving complex problems of modeling nonlinear dependencies, taking into account the dynamic components. More accurate forecasting and management allows for more accurate management decisions, which helps to reduce the risks of irrigated agriculture and the sustainability of agricultural production in areas of risky farming.

\section{References}

1) The State of Food and Agriculture 2020. Overcoming water challenges in agriculture, (2020), 210p .

2) G. Olgarenko, Land Reclamation and Water Management, 1, 44-47 (2018)

3) A. McCarthy, N. Hancock, S. Raine, Comput. Electron. Agric, 101, 135-147 (2014) doi:

10.1016/j.compag.2013.12.004 
4) H. Navaro-Hellin, J. Martinez-del-Ricon, R. Domingo-Miguel, F. Soto-Valles, R. Torres-Sanchez, Comput. Electron. Agric, 124, 121-131 (2016) doi: 10.1016/j.compag.2016.04.003

5) E. Giusti, S. Marsili -Libelli, Environ. Model. Softw., 63, 73-86 (2015) doi: 10.1016/j.envsoft.2014.09.020

6) S. Rasp, M. Pritchard, P. Gentine, Proc. Natl. Acad. Sci. USA, 115, 9684-9689 (2018) doi: $10.1073 /$ pnas. 1810286115

7) F. Sévellec, S. S. Drijfhout, Nat. Commun. 9 (2018) doi: 10.1038/s41467-018-05442-8.

8) Yu.F. Kurdyukov, M. Yu. Vasilieva, N.G. Levitskaya, G.A. Kulikova, Grain industry of Russia, 3, 58-62 (2014)

9) V. Meshcheryakov, P. Saraev, O. Meshcheryakova, Management Problems, 6, 71-73 (2013)

10) M. Belov, I. Nosirov, C. Fyong, Izvestia SPbGETU 'LETI",4, 73-80 (2018)

11) S. Khaikin, Neural Networks: A Complete Course, 2nd ed. (2019), 1103 p.

12) J. Schmidhuber, Neural Network, 61, 85-117(2015)

13) G.F. Ivanova, N.G. Levitskaya, I.A. Orlova, Izvesti Saratov University. New series. Earth sciences, 13(2), 10-12 (2013)

14) Roshydromet. Federal Service for Hydrometeorology and Environmental Monitoring, Electronic resource - Access Mode: http://www.meteorf.ru/

15) V. Dyakonov, MATLAB. Complete tutorial, (2012), 768 p.

16) G. Kamyshova, D. Solovyev, S. Zatinatsky, D. Kolganov, N. Terekhova, E3S Web of Conferences 175, 05007 (2020)

17) T. Ise, Y. Oba, Front. Robot. AI, 6, 6-32 (2019) doi: 10.3389/frobt.2019.00032

18) M. Beale, M. Hagan, H. Demuth, Neural Network ToolboxTM User's Guide (The Math Works, Inc, 2015) 